\title{
Evaluating cervical cancer with magnetic resonance imaging (MRI): a pictorial review
}

\author{
S D Perera ${ }^{1}$ \\ Sri Lanka Journal of Obstetrics and Gynaecology 2009; 31: 55-58
}

Key words: cervical cancer evaluation, magnetic resonance imaging.

\section{Introduction}

Cervical cancer is the $3 \mathrm{rd}$ commonest female malignancy worldwide. There is a high incidence in the developing countries and in low socio economic groups. The cancer staging is very important in determining the treatment and prognosis. Clinical staging is often inaccurate. MRI is increasingly used for initial staging and follow up. MRI with its ability demonstrates soft tissues in greater detail and to some extent characterise different types of soft tissues, is superior to ultrasound (trans abdominal and trans vaginal) and computed tomography. For MRI the accuracy of local staging is reported between $81 \%$ $91 \%$. For staging nodes the reported accuracy is $85 \%$ $-93 \%{ }^{1,2}$

\section{Patterns of spread}

$90 \%$ of cervical cancers are squamous cell carcinomas. They arise from the squamo columnar junction in the endocervical canal. Initially the tumour infiltrates the cervical stroma spreading laterally in to the parametrium and then to the pelvic sidewall. The ureters which are in close proximity to the cervix can become invaded and obstructed. The tumour may spread superiorly to the uterus and inferiorly to involve the vagina. Anterior spread in to the anterior parametrium would involve the bladder and posterior infiltration would involve the utero sacral ligaments, rectum and the pelvic floor.

Lymph node spread is initially to the para cervical and parametrial nodes. The obturator nodes are the next to be involved with pre sacral and peri rectal nodes. The tumour could then spread to the internal iliac, external iliac, common iliac and retroperitoneal nodes.

This pattern of spread can be clearly identified on MR imaging (Figure 6). Uncommonly the tumour can spread to liver, lung and bone.

\footnotetext{
${ }^{1}$ Consultant Radiologist, Department of Radiology, Southend University Hospital NHS Foundation Trust, Westcliff-on-Sea, UK.

E-mail: sdperera@hotmail.com
}

\section{Normal and abnormal cervix on MRI}

Normal cervix on MRI (Figures 1 \& 2 ) on a T2W image shows a bright endocervical canal surrounded by mucosa which is slightly less bright. Around the mucosa is a dark area depicting the predominantly fibrous part of the cervical stroma. The outer part of the cervix returns a higher signal being composed predominantly of smooth muscle. Beyond this is the parametrium shown as tissue with brightness close to fatty tissue, being composed of fat, lymphatics and blood vessels ${ }^{3}$.

Early stage cervical cancer is shown as an area of intermediate signal often within or close to the endocervical canal (Figure 3). As the tumour grows it expands within the dark stomal ring before breeching this structure to involve the outer cervix and parametrium (Figures 4 \& 5). Parametrial involvement begins frequently as spiculations or stands of intermediate signal material extending out into the fat (Figures $5 \& 6$ ).

Breach of the dark stromal ring in on an axial image of the cervix is a useful sign of parametrial invasion $^{3}$. As tumour spreads laterally towards the pelvic side wall, involvement of the ureters can occur with ureteric obstruction and hydronephrosis (Figure $7)$. The obstructed ureter shows a bright circular structure above the level of obstruction (Figure 6).

Spread of tumour along the utero sacral ligament is a common observation in advanced cervical cancer. Tumour can then spreads to the pelvic sidewall and involve the piriformis muscle and the pelvic floor (Figure 9).

Involvement of the body of the uterus is depicted by intermediate signal material causing expansion of the uterus destroying its normal zonal structure (Figure 8). Often there is retained fluid within the endometrial cavity due to cervical or lower segment obstruction. Infiltration anteriorly through the anterior parametrium leads to bladder involvement as shown by intermediate signal tumour occupying the normal, low signal wall of the bladder (Figure 8).

Occasionally there will be tumour arising from adjacent organs which may infiltrate the cervix rather than the tumour arising from the cervix. The commonest scenario is when tumours from the uterine 
body invade the cervix. It not uncommon for advanced bladder tumours and rectal tumours to infiltrate widely in the pelvis including the cervix and body of uterus (Figures 10, 11).

Once the cervical tumour is imaged and staged with MRI, its utility does not end. MRI is an excellent way of monitoring surgical as well as non surgical treatment with chemo radiation. If chemo radiation is successful reconstitution of the cervix can be seen. This may take several months to a year. The uterus and cervix shrinks and becomes dark with loss of the normal zonal anatomy (Figures 12, 13). Tumour recurrence can also be monitored and evaluated with $\mathrm{MRI}^{4,5}$ (Figure 9).

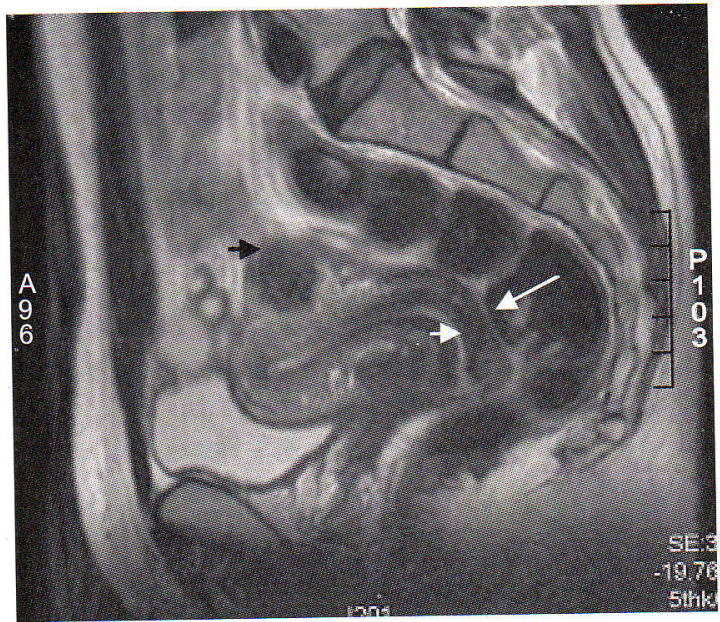

Figure 1. Sagittal T2W MR scans of pelvis. Normal cervix (long white arrow), endocervical canal (short white arrow). Note fibroid (black arrow).

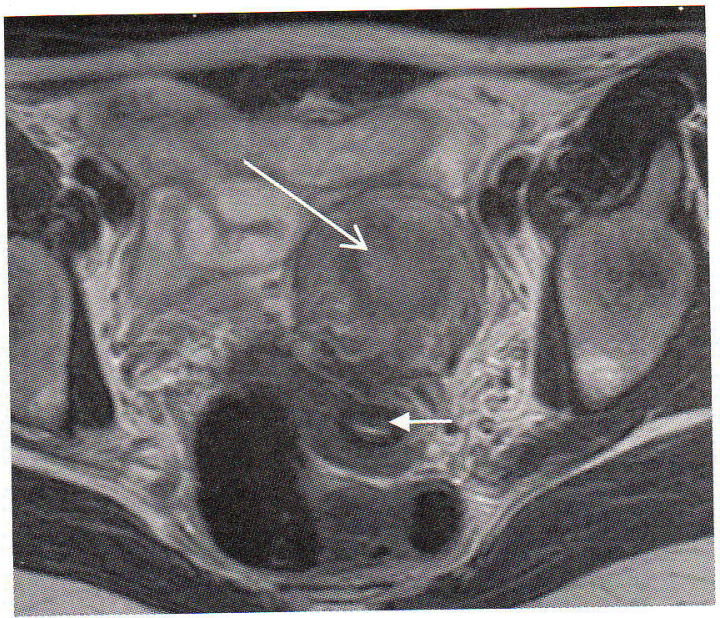

Figure 2. Axial T2W MR pelvic scan. Fibrous cervical stroma shown as a black ring surrounding a bright endocervical canal (short white arrow). Endometrium (long white arrow).

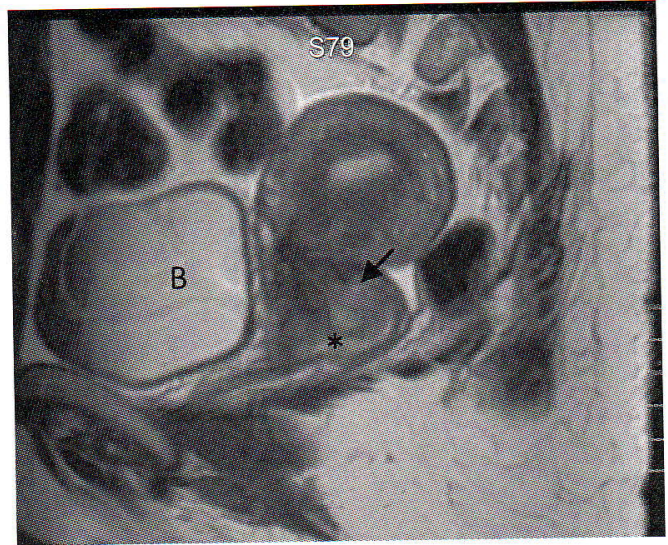

Figure 3. Sagittal MR scan showing Tumour confined to cervix (black arrow). Endocervical canal (asterix). Bladder (B).

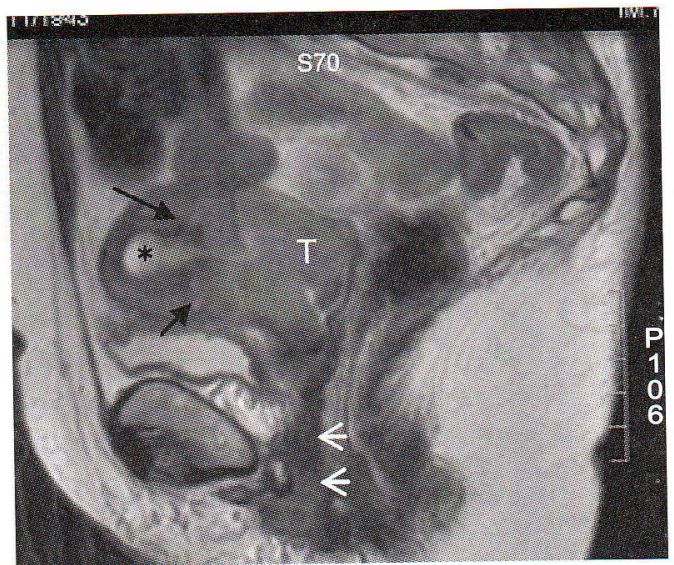

Figure 4. Bulky cervical tumour (T) extending up into the uterine body (black arrows). There is fluid in a distended endometrial cavity (asterix) due to obstruction of the endocervical canal. The vaginal wall is thickened and likely to be involved by tumour (white arrows).

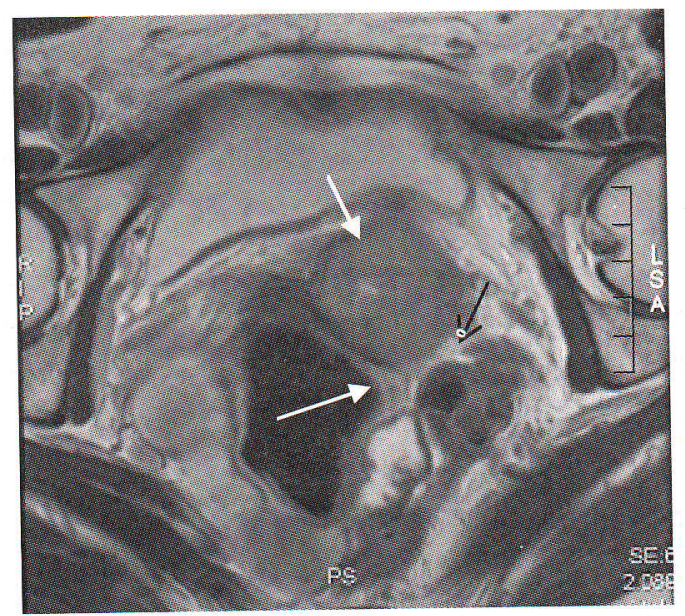

Figure 5. Same patient as above showing left parametrial invasion with strands extending into the pericervical fatty tissue (black arrow). Note the disruption of black ring of normal cervical stroma (white arrows).

Sri Lanka Journal of Obstetrics and Gynaecology 


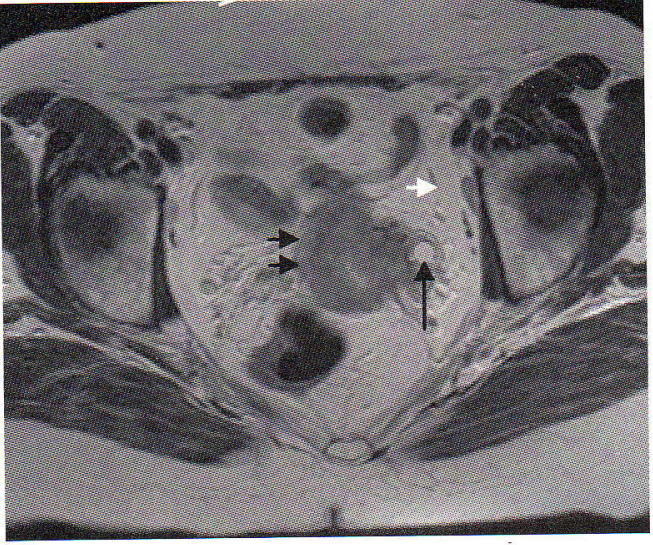

Figure 6. Tumour (double arrow heads) invading the left parametrium encasing and obstructing the left ureter (white circle). Note left obturator lymph node having same signal intensity of the main tumour (white arrow) and likely to be involved.

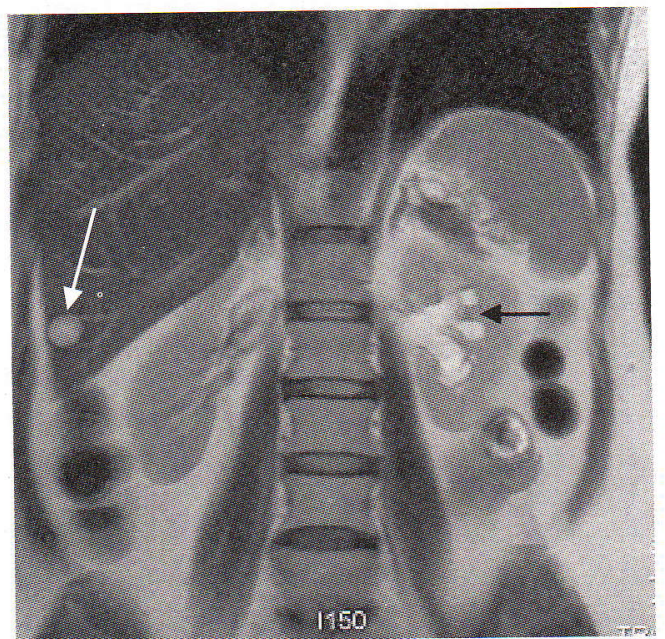

Figure 7. Same patient as figure 6 showing left kidney hydronephrosis (black arrow). Incidental liver cyst (white arrow).

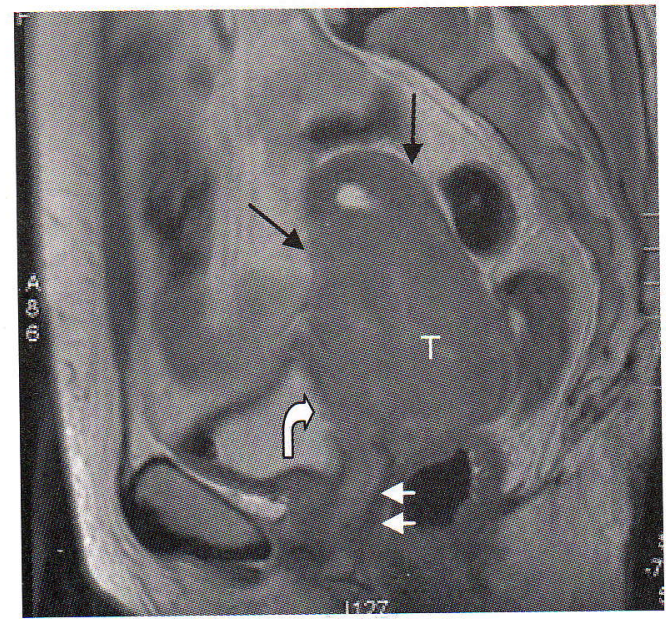

Figure 8. Very large cervical tumour (T) invading uterine body (black arrows) and the vagina down to its lower third (white arrow heads). Note, the posterior wall of the bladder is ill defined and suggests bladder invasion (curved white arrow).

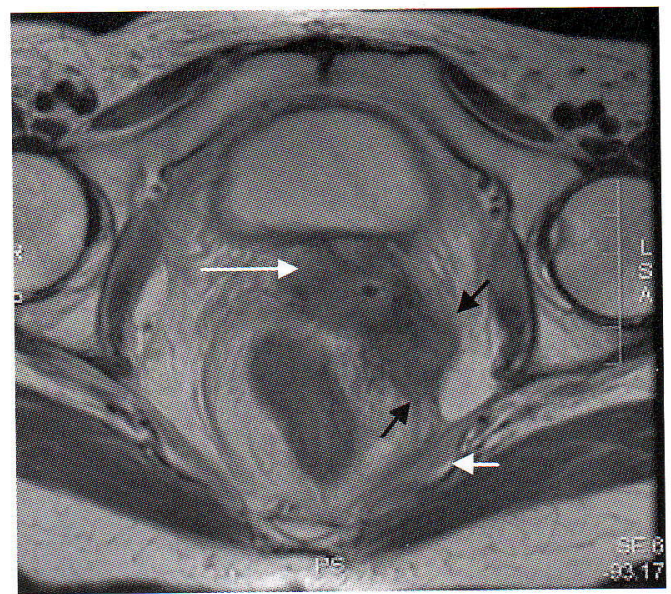

Figure 9. Recurrent cervical tumour following trachelectomy for stage $1 \mathrm{~b}$ disease. Now tumour is seen to extend posteriorly along the left utero sacral ligament (black arrows) towards the piriformis muscle (white arrowhead). Tumour is extending anteriorly towards the bladder but has not actually invaded the bladder wall (white arrow).

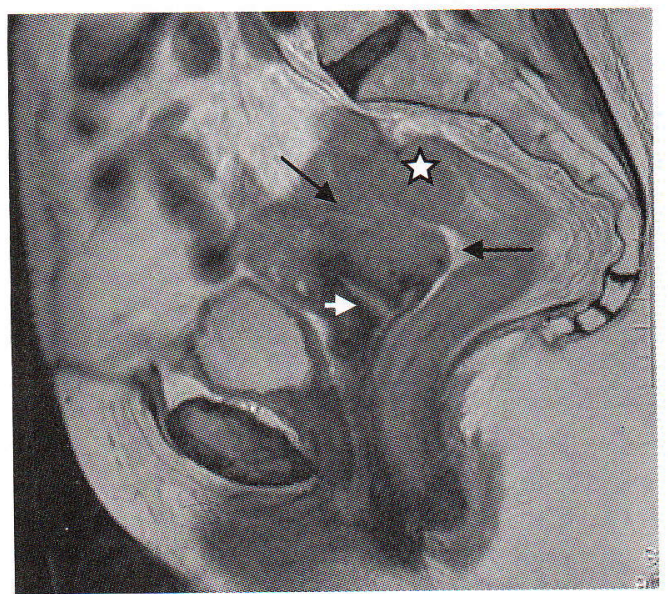

Figure 10. There is a mass in the rectum (star) which is extending anteriorly to involve the posterior cervix (black arrows). Note, the endocervical canal is normal (short white arrow).

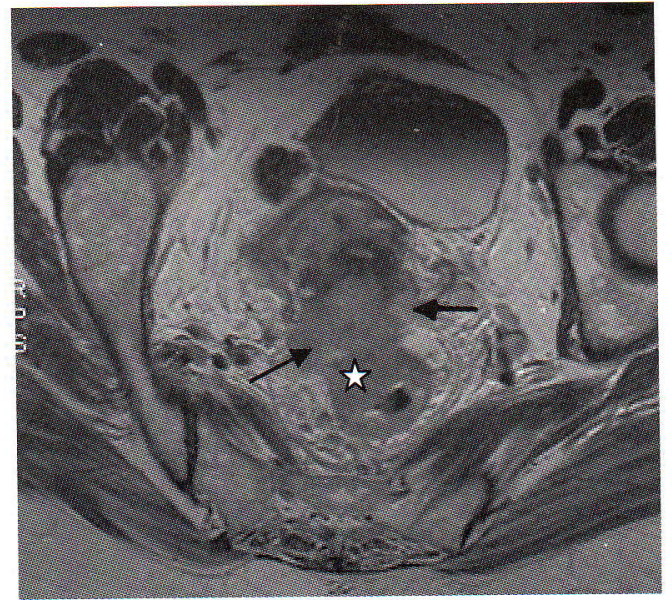

Figure 11. Axial MR scan of the same patient as above showing the rectal mass (star) growing into the posterior cervix (arrows). 


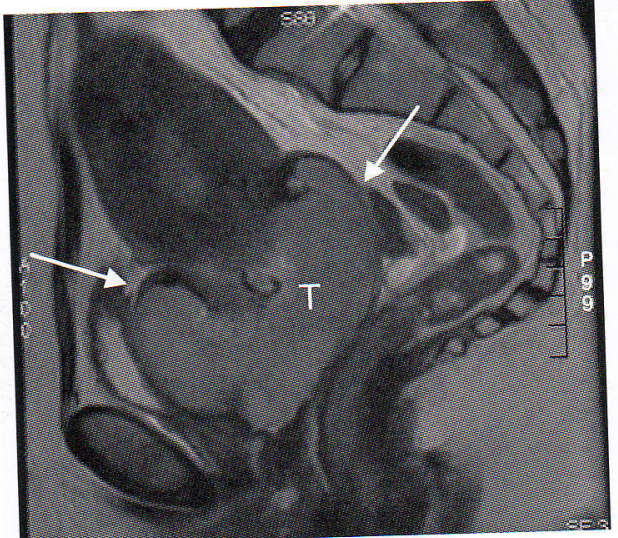

Figure 12. Large cervical tumour $(\mathrm{T})$ protruding into the upper vagina (white arrows) distending the vaginal fornices but not invading it.

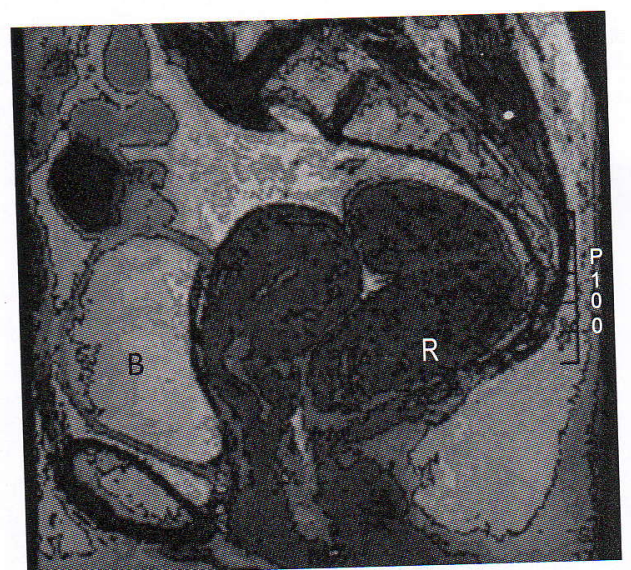

Figure 13. Same patient as Fig 12. after chemotherapy and radiation treatment (14 months between scans). Note that the cervix has reconstituted and has become almost uniformly black. The cervix and uterus has lost its zonal structure. Bladder (B) Rectum (R).

\section{Conclusion}

MRI is a very useful tool for accurate staging of cervical cancer. It is used both for local staging and evaluation of local and distant nodal metastases. MRI is superior to clinical staging and help guide the optimum treatment whether it is surgical or chemo radiation. It can demonstrate pelvic pathology arising outside the uterus accurately. MRI is also a valuable tool in evaluating the effect of treatment and recurrences after treatment.

\section{References}

1. Hulse PA, Carrington M (2004). MRI Manual of Pelvic Cancer. London: Martin Dunitz. p45-75

2. Hricak $\mathrm{H}$, Gatsonis $\mathrm{C}$, Coakley FV, et al. Early Invasive Cervical Cancer: CT and MR Imaging in Preoperative Evaluation-ACRIN/GOG Comparative Study of Diagnostic Performance and Interobserver Variability. Radiology 2007; 245: 491-8.

3. Nicolet V, Carignan L, Bourdon F, Prosmanne O. MR imaging of cervical carcinoma: a practical staging approach. Radio Graphics 2000; 20: 1539-49.

4. Jeong YY, Kang HK, Chung TW, Seo JJ, Park JG. Uterine cervical carcinoma after therapy: CT and MR imaging findings. Radio Graphics 2003; 23: 969-81.

5. Flueckiger F, Ebner F, Poschauko H, et al. Cervical cancer: serial MR imaging before and after primary radiation therapy - a 2-year follow-up study. Radiology 1992; 184: 89. 